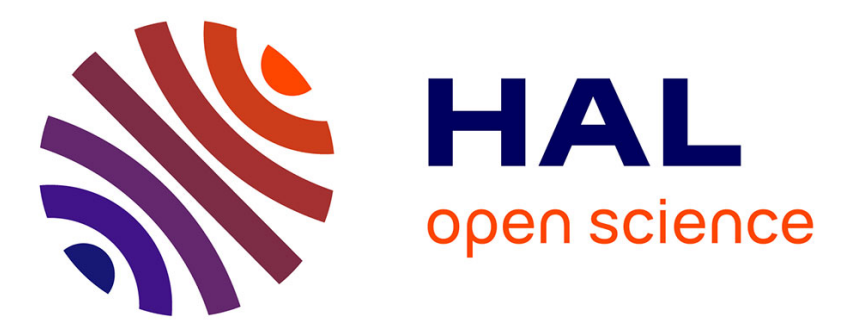

\title{
Renormalization group evaluation of the second virial coefficient of polydisperse polymer solutions
}

\author{
S. Stepanow, E. Straube
}

\section{To cite this version:}

S. Stepanow, E. Straube. Renormalization group evaluation of the second virial coefficient of polydisperse polymer solutions. Journal de Physique Lettres, 1985, 46 (23), pp.1115-1122. 10.1051/jphyslet:0198500460230111500 . jpa-00232945

\section{HAL Id: jpa-00232945 https://hal.science/jpa-00232945}

Submitted on 1 Jan 1985

HAL is a multi-disciplinary open access archive for the deposit and dissemination of scientific research documents, whether they are published or not. The documents may come from teaching and research institutions in France or abroad, or from public or private research centers.
L'archive ouverte pluridisciplinaire HAL, est destinée au dépôt et à la diffusion de documents scientifiques de niveau recherche, publiés ou non, émanant des établissements d'enseignement et de recherche français ou étrangers, des laboratoires publics ou privés. 
Classification

Physics Abstracts

$36.20-61.40 \mathrm{~K}-82.70$

\title{
Renormalization group evaluation of the second virial coefficient of polydisperse polymer solutions
}

\author{
S. Stepanow and E. Straube \\ Technische Hochschule "Carl Schorlemmer " Leuna-Merseburg, Sektion Physik, \\ DDR-4200 Merseburg, Otto-Nuschke-Str., G.D.R.
}

(Reçu le 14 janvier 1985, révisé le 12 août, accepté le 14 octobre 1985)

\begin{abstract}
Résumé. - A l'aide du groupe de renormalisation on a évalué le deuxième coefficient du viriel d'une solution composée de deux polymères monodispersés. On a montré que le terme croisé $A_{12}$ atteint sa valeur limite lorsque le poids moléculaire de l'un des deux polymères tend vers l'infini. Un tel comportement donne le maximum de $A_{2}$ avec le changement en teneur relative des polymères. Le maximum calculé au premier ordre du groupe de renormalisation est situé en dehors du domaine expérimental.
\end{abstract}

\begin{abstract}
The second virial coefficient $A_{2}$ for a mixture of two monodisperse polymers is studied by means of the renormalization group. It is found that the cross-term $A_{12}$ has a finite limit if the segment number of one of the polymers tends to infinity. Such behaviour predicts a maximum of $A_{2}$ in dependence on the relative composition of the polymers. The maximum resulting from our firstorder RG-calculation lies outside the molecular weight ratio investigated so far experimentally.
\end{abstract}

\section{Introduction.}

In recent years increasing attention has been paid to the second virial coefficient of a solution of two different polymers [1-10]. A number of authors applied the renormalization group (RG) methods to this problem [2-4, 6-10]. Most of these authors used the massless renormalization schemes which are successful by the study of the monodisperse polymer solutions. The characteristic feature of the massless schemes is that the differential equations of the RG of the quantities under consideration are independent of the degrees of polymerization of both chains $N_{1}$ and $N_{2}$. The dependence on $N_{1}$ and $N_{2}$, which governs the scaling behaviour of the physical quantities, is lost in the RG equations, and the massless schemes are inefficient for the study of the polydispersity effects as will be outlined in detail in this paper. In this paper in order to study the polydispersity effects, we start from the massless scheme and carry out the renormalization at finite degrees of polymerization of the chains. The method used here is the quantum field theoretic renormalization group [11] formulated in terms of the polymer correlation functions [12]. Section 2 briefly introduces our regularization method. Section 3 contains the RG evaluation of the effective interaction constants. Section 4 gives the evaluation of the second virial coefficient. Section 5 gives the comparison with the massless schemes. 


\section{Regularization formalism.}

The osmotic second virial coefficient of a mixture of two polymer samples of different molecular weight is given by

$$
A_{2}=A_{11} w_{1}^{2}+2 A_{12} w_{1} w_{2}+A_{22} w_{2}^{2}
$$

$w_{i}$ is the weight fraction of polymer $i$. The coefficients $A_{i j}$ can be expressed as follows by the Fourier transformation of the connected end-to-end chain correlation functions

$$
G_{m, n}^{\mathrm{c}}\left(p_{1}, \ldots, p_{2 m+2 n}\right)
$$

with prefactors $(2 \pi)^{d} \delta^{d}\left(p_{1}+\cdots+p_{2 m+2 n}\right)$ removed and external momenta equal to zero [12-14]

$$
A_{11}=-\frac{1}{2}\left(N_{\mathrm{A}} / M_{1}^{2}\right) G_{2,0}^{\mathrm{c}}(0) / G_{1,0}^{2}(0) ; \quad A_{12}=-N_{\mathrm{A}} /\left(M_{1} M_{2}\right) G_{1,1}^{\mathrm{c}}(0) /\left(G_{1,0}(0) G_{0,1}(0)\right)
$$

and analogously for $A_{22} . M_{i}$ is the molecular weight of polymer $i$ and $N_{\mathrm{A}}$ is the Avogadro number. The indices $m$ and $n$ denote the number of both chains involved in the correlation function. The examples of diagrams contributing to $G_{1,1}^{\mathrm{c}}\left(p_{1}, \ldots, p_{4}\right)$ are shown in figure 1 .

The continuous model of polymer chains which describes the real polymers on the long scale breaks down on a scale comparable to the statistical segment length $l$. This will be taken into account by limiting the integration over the internal momenta in the perturbation expansions (see [12, 14]) of physical quantities at the upper limit, by a cutoff $\Lambda \sim 1 / l$. The renormalizability of the polymer theory enables an absorption of the ultraviolet singularities, which appear in four dimensions as logarithms $\ln ^{k}\left(L l \Lambda^{2}\right)$ ( $L$ is the contour length of the chain) and in $d$ dimensions as poles $1 / \varepsilon^{k}(\varepsilon=4-d)$, in the parameters of the theory. It is well known [11] that the ultraviolet singularities appearing in a renormalizable theory are expressed by the singularities of a finite type of diagrams. If one wants to apply the regularization methods of the quantum field theory to the polymer correlation functions, one must, at first, study these diagrams. An analysis of the divergences of the polymer CF carried out in the same way as in the field theory [11] shows that the divergences of the polymer CF can be expressed by the divergences of the selfenergy $\Sigma_{i}(p)$ and the vertex $\Gamma_{i j}\left(p_{1}, \ldots, p_{4}\right)$ defined for the polymer diagrams as follows

and

$$
\tilde{G}_{1,0}(p)=\exp \left(-p^{2} S_{1}^{0} / 2 d\right) \Sigma(p)
$$

$$
\widetilde{G}_{1,1}^{\mathrm{c}}\left(p_{1}, \ldots, p_{4}\right)=-v_{12}^{0} S_{1}^{0} S_{2}^{0} \exp \left(-p_{1}^{2} S_{1}^{0} / 2 d-p_{2}^{2} S_{2}^{0} / 2 d\right) \Gamma_{12}\left(p_{1}, \ldots, p_{4}\right)
$$

where $S_{i}^{0}=L_{i} l$ and $G_{1,1}^{\mathrm{c}}(p)$ and $\widetilde{G}_{1,1}^{\mathrm{c}}\left(p_{1}, \ldots, p_{4}\right)$ denote the set of $1 p$-irreducible diagrams contributing to the $\mathrm{CF} G_{1,0}(p)$ and $G_{1,1}^{\mathrm{c}}$. It is important that the singularities appearing in the perturbation expansions are expressed by those of the $\Sigma_{i}(0)\left(\partial \Sigma_{i}(p) / \partial p^{2}\right)_{p=0}$ and $\Gamma_{i j}(0)$. After illustrating the character of the divergences of the polymer CF the elimination of the divergences can be
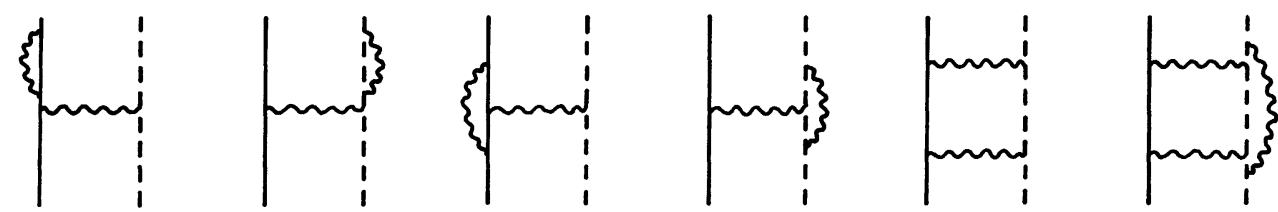

Fig. 1. - Diagrams contributing to $G_{1,1}^{\mathrm{c}}\left(p_{1}, \ldots, p_{4}\right)$. 
carried out in the same way as in the field theory (see[11], Chap. 6) and the result is

$$
\begin{gathered}
G_{m, n}^{\mathrm{c}}\left(p_{1}, \ldots, p_{2 m+2 n} ; S_{i}^{0}, v_{i j}^{0}, \Lambda\right)=\exp \left(m S_{1} \Delta \Sigma_{1}+n S_{2} \Delta \Sigma_{2}\right) . \\
X_{1}^{m} X_{2}^{n} G_{m, n}^{\mathrm{c}}\left(p_{1}, \ldots, p_{2 m+2 n} ; S_{i}, v_{i j}\right)_{\mathrm{reg}}
\end{gathered}
$$

The connection between the unregularized and regularized CF like (3) was proposed for the first time by des Cloizeaux [14]. The $R$-operation by Bogoliubov [11] (for the polymer case see [12]) provides the method for constructing the regularized $C F$ on the right-hand side of equation (3). $\Delta \Sigma_{i}$ contains the « masses » divergences which do not contribute to the physical quantities [15], $v_{i j}^{0}$ are the bare excluded volume strengths. The effective quantities $S_{i}$ and $v_{i j}$ appearing in the regularized theory, may be expressed by the counterterms $X_{i}, Z_{3}^{i}, Z_{1}^{i}$ and $Z_{12}$ in the following way [12]

and

$$
S_{i}=X_{i} Z_{3}^{i} S_{i}^{0}, v_{i i}=v_{i i}^{0} Z_{1}^{i} /\left(Z_{3}^{i}\right)^{2}
$$

$$
v_{12}=v_{12}^{0} Z_{12} /\left(Z_{3}^{1} Z_{3}^{2}\right) \text {. }
$$

The counterterms $X_{i}, Z_{3}^{i}, Z_{1}^{i}$ and $Z_{12}$ are expressed by the self-energy $\Sigma_{i}(p)$ and the vertex $\Gamma_{i j}(0)$ as follows [12]

$$
\begin{gathered}
X_{i}=1 /\left(1-\Sigma_{i}(0)\right), Z_{3}^{i}=1 / X_{i}-\left(S_{i}^{0}\right)^{-1}\left(\partial \Sigma_{i} / \partial p^{2}\right)_{p=0} \\
Z_{1}^{i}=1+\Gamma_{11}(0) \text { and } Z_{12}=1+\Gamma_{12}(0) .
\end{gathered}
$$

By using the $\Lambda^{2}$-scheme (massless renormalization scheme) only the ultraviolet diverging parts of the selfenergy and the vertex are involved as counterterms in the subtraction procedure. In the monodisperse case the $\Lambda^{2}$-scheme leads to simple RG equations for the effective quantities $S_{i}$ and $v_{i j}$. A special choice of the parameter of RG (matching condition) allows one to absorb the infrared singularities (causing the scaling behaviour) in the parameter $S_{i}$ and $v_{i i}$ [2]. In the polydisperse case the $\Lambda^{2}$-scheme is inefficient. Whereas the ultraviolet behaviour of the polydisperse diagrams is the same as for the monodisperse ones, the infrared behaviour is governed by two parameters $N_{1}$ and $N_{2}$. Therefore, RG equations appearing in the massless scheme cannot reproduce the infrared singularities of the perturbation expansions. Consequently, in the polydisperse case, we use the complete vertex $\Gamma_{i j}(0)$ (with integrals cut at $\Lambda$ ) in the counterterms of the regularization procedure. The selfenergy-like singularities can be treated as previously in the monodisperse case.

\section{The renormalization group.}

The key quantity in equation (3) containing the polydispersity effects is the effective interaction constant $v_{12} \cdot v_{12}(\Lambda)$ is given by (3) as a series in powers of $v_{i j}^{0}$. Applying the subtraction procedure to $\Gamma_{12}(\Lambda) \equiv \Gamma_{12}(p=0, \Lambda)$ by using $\Gamma_{i j}\left(\Lambda^{\prime}\right)\left(\Lambda^{\prime}<\Lambda\right)$ as counterterms, we can express $v_{12}(\Lambda)$ by $v_{i j}\left(\Lambda^{\prime}\right)$

$$
v_{12}(\Lambda)=v_{12}\left(\Lambda^{\prime}\right)\left(1+\tilde{\Gamma}_{12}\left(v_{i j}\left(\Lambda^{\prime}\right), \Lambda^{\prime}, \Lambda\right)\right) .
$$

Both $v_{12}(\Lambda)$ and $v_{12}\left(\Lambda^{\prime}\right)$ depend on the effective parameter $S_{i}$. The contributions to $\Gamma_{12}$ up to $\mathcal{O}\left(v_{i j}\right)$ as well as the contributions to $\widetilde{\Gamma}_{12}$ result from the last three one-loop diagrams in figure 1 . The equation (5) may be obtained in the following way, too. The equation (4) may be interpreted as an equation for $v_{12}\left(\Lambda^{\prime}\right)$. Inverting $v_{i j}\left(\Lambda^{\prime}\right) v_{i j}^{0}$ are obtained as functions of $v_{i j}\left(\Lambda^{\prime}\right)$ which we substitute in (4). Then $v_{12}(\Lambda)$ is obtained as a function of $v_{i j}\left(\Lambda^{\prime}\right)$. This second method of obtained equation (5) resembles the renormalization method by des Cloizeaux [15]. In the following $\widetilde{\Gamma}_{12}$ is approximated by the contributions from the one-loop diagrams shown in figure 1 . Differentia- 
ting (5) with respect to $\Lambda$ and setting $\Lambda=\Lambda^{\prime}$ we get

$$
\begin{aligned}
& \begin{aligned}
\partial v_{12} / \partial x=-\frac{1}{2}(2 d)^{d / 2} S_{d} x^{-2-\varepsilon / 2} v_{12} & {\left[2 v_{12}\left(S_{1} S_{2} x\right)^{-1} \times\right.} \\
& \left.\times W\left(x S_{1}, x S_{2}\right)+v_{11} S_{1}^{-1} Y\left(x S_{1}\right)+v_{22} S_{2}^{-1} Y\left(x S_{2}\right)\right]
\end{aligned} \\
& \text { where }
\end{aligned}
$$

$x=\Lambda^{2}, Y(x)=x+(2+x) \exp (-x)-2$,

$$
W(x, y)=(x+\exp (-x)-1)(y+\exp (-y)-1) \text { and } S_{d}=2 /(4 \pi)^{d / 2} \Gamma(d / 2) .
$$

The equation for $v_{i i}$ follows from (6) putting $S_{1}=S_{2}=S_{i}$ and $v_{12}=v_{11}=v_{22}=v_{i i}$. If one approximates $Y\left(x S_{i}\right)$ and $W\left(x S_{1}, x S_{2}\right)$ in (6) by their asymptotic forms one will obtain the equation for $v_{12}$ in the massless scheme

$$
\partial v_{12} / \partial x=-\frac{1}{2}(2 d)^{d / 2} S_{d} x^{-1-\varepsilon / 2} v_{12}\left(2 v_{12}+v_{11}+v_{22}\right)
$$

For $S_{i}$ the following equation was obtained in the one-loop approximation of the renormalization group [12]

$$
S_{i}=S_{i}^{0}\left(1+\frac{8}{\varepsilon}(d / 2 \pi)^{d / 2} v_{i i}^{0} l^{-4} S_{i}^{\varepsilon / 2}\right)^{1 / 4} .
$$

It is easy to see that in the scaling region, when the second term in the bracket dominates and $\varepsilon$ is small, the preceding equation produces the scaling behaviour of $S_{i}$

$$
S_{i}=\left(\frac{8}{\varepsilon}(d / 2 \pi)^{d / 2} v_{i i}^{0} l^{-4}\right)^{\frac{2}{\varepsilon}(2 v-1)} S_{i}^{02 v}
$$

with $v=1 / 2+\varepsilon / 16$.

The solution of equation (6) with the boundary conditions

is

$$
\lim _{x \rightarrow \infty} v_{12}(x)=v_{12} \text { and } \lim _{x \rightarrow 0} v_{12}(x)=v_{12}^{0}
$$

$$
\begin{aligned}
v_{12}(x)=v_{12}^{0} \mu(x) /\left(1+v_{12}^{0} l^{-4}(d / 2 \pi)^{d / 2} 2 / \Gamma(d / 2)\right. & \times \\
& \left.\times \int_{0}^{x} \mathrm{~d} t t^{-3-\varepsilon / 2}\left(S_{1} S_{2}\right)^{-1} \mu(t) W\left(t S_{1}, t S_{2}\right)\right)
\end{aligned}
$$

where

$$
\begin{aligned}
\mu(x)=\exp \left[-l^{-4}(d / 2 \pi)^{d / 2} / \Gamma(d / 2)\right. & \times \\
& \left.\times \int_{0}^{x} \mathrm{~d} t t^{-2-\varepsilon / 2}\left(S_{1}^{-1} v_{11}(t) Y\left(t S_{1}\right)+S_{2}^{-1} v_{22}(t) Y\left(t S_{2}\right)\right)\right] .
\end{aligned}
$$

The $v_{i i}(x)$ are obtained by putting in (8) $S_{1}=S_{2}=S_{i}$ and $v_{12}^{0}=v_{11}^{0}=v_{22}^{0}=v_{i i}^{0}$

$$
\begin{aligned}
v_{i i}(x)=v_{i i}^{0}\left\{1+v_{i i}^{0} l^{-4}(d / 2 \pi)^{d / 2}\right. & 2 / \Gamma(d / 2) \times \\
\times & \left.\int_{0}^{x} \mathrm{~d} t t^{-2-\varepsilon / 2}\left[S_{i}^{-1} Y\left(t S_{i}\right)+t^{-1} S_{i}^{-2} W\left(t S_{i}, t S_{i}\right)\right]\right\} .
\end{aligned}
$$


Equations (8) to (10), in addition to the singular parts, contain also the regular ones which can now be eliminated. In order to make this we approximate $Y$ and $W$ in (10) by their asymptotic parts $Y(x S) \approx x S$ and $W(x S, x S) \approx(x S)^{2}$. Then the integral in (10) becomes diverging at the lower limit. In order to make it finite and reproduce its infrared singularity in the vicinity of four dimensions the cutoff $\Lambda_{\min }^{i}=1 / S_{i}$ has to be introduced. Instead of $(10)$ we arrive at

$$
v_{i i}(x)=v_{i i}^{0} /\left(1+4 v_{i i}^{0} l^{-4}(d / 2 \pi)^{d / 2} \frac{2}{\varepsilon}\left(S_{i}^{\varepsilon / 2}-x^{-\varepsilon / 2}\right)\right) .
$$

In the scaling region $v_{i i}$ behaves as $v_{i i} \sim S_{i}^{-\varepsilon / 2}$. In the same way for $\mu(x)$ follows

$$
\mu(x)=\left(v_{11}(x) / v_{11}^{0}\right)^{1 / 4}\left(v_{22}(x) / v_{22}^{0}\right)^{1 / 4} .
$$

Now we look at the integral in equation (8). Because the infrared singularity of this integral is controlled by the short chain (henceforth the chain 1), the lower cutoff in this integral has to be identified with $\Lambda_{\min }^{1}=1 / S_{1}$. The result for $v_{12}$ is

$$
v_{12}=v_{12}^{0}\left(S_{1}^{0} / S_{1}\right)\left(S_{2}^{0} / S_{2}\right) / Q
$$

where $Q$ is given by

$$
Q=1+2 v_{12}^{0} l^{-4}(d / 2 \pi)^{d / 2} \int_{S_{1}^{-1}}^{\infty} \mathrm{d} t t^{-1-\varepsilon / 3} \mu(t) .
$$

An equation similar to (13) was earlier obtained in reference [4] in the framework of polymermagnet analogy. By using the massless scheme (solution of Eq. (7)) we would obtain an expression similar to (13), with the difference that instead of two cutoffs $\Lambda_{\min }^{1}=1 / S_{1}$ and $\Lambda_{\min }^{2}=1 / S_{2}$ there would only appear one cutoff $\Lambda_{\min }$. Such an expression for the interaction constant $v_{12}$ was used in [3] by using the polymer-magnet analogy.

\section{The second virial coefficient.}

Now we turn to the evaluation of the second virial coefficient which has to be performed by means of equations (1) and (3). Instead of $A_{12}$ we consider the function $h_{12}$ defined by

$$
A_{12}=N_{\mathrm{A}} /\left(M_{\mathrm{s}}^{1} M_{\mathrm{s}}^{2}\right) v_{12}^{0} h_{12}\left(z_{1}, N_{2} / N_{1}\right)
$$

where $z_{1}=(d / 2 \pi)^{d / 2} v_{11}^{0} l^{-4}\left(S_{1}^{0}\right)^{\varepsilon / 2}$. Evaluating the regular part of the fifth diagram in figure 1 in four dimensions we obtain

$$
h_{12}=Q^{-1}\left\{1+\left(v_{12} / v_{11}\right) g_{11}\left(t^{-1} \ln (1+t)+t \ln ((1+t) / t)+2 \ln ((1+t) / t)-1\right)\right\}
$$

where $t=S_{2} / S_{1}$, and the dimensionless interaction constant

$$
g_{11}=z_{1} v_{11} / v_{11}^{0}\left(S_{1} / S_{1}^{0}\right)^{\varepsilon / 2}
$$

is introduced. For large $S_{1}, g_{11}$ tends to the fixed point $g^{*}=\varepsilon / 8$. The function $h_{i i}\left(z_{i}\right)$ for the monodisperse solution is obtained by (16) putting $N_{1}=N_{2}=N_{i}$ and $v_{12}^{0}=v_{11}^{0}=v_{22}^{0}=v_{i i}^{0}$

$$
h_{i i}=v_{i i} / v_{i i}^{0}\left(S_{i} / S_{i}^{0}\right)^{2}\left(1+g_{i i}(4 \ln 2-1)\right) .
$$

In the scaling region $h_{i i}$ scales as $N_{i}^{v d-2}$. For large $N_{1}$ and $N_{2} \sim N_{1} h_{12}\left(z_{1}, N_{2} / N_{1}\right)$ also scales as $N_{1}^{v d-2} \varphi\left(N_{2} / N_{1}\right)$, where $\varphi(x)$ is given by (14) and (16). For $N_{2} \rightarrow \infty$ and $N_{1}$ finite equation(16) 
gives

$$
h_{12}\left(z_{1}, \infty\right)=1 .
$$

That means that for given $z_{1}$ and $N_{1}$ there exists a $N_{2}^{*}$ such that for $N_{2}>N_{2}^{*}$

$$
h_{22}<h_{11}<h_{12}
$$

holds. Equation (20) is the condition for the appearence of the maximum of the second virial coefficient $A_{2}$ with variation of the solute composition [16]. Figure 2 shows $z_{2}^{*} / z_{1} v s . z_{1}$ obtained from the equations (16) and (18). Our result agrees qualitatively with that of Tanaka and Šolc [5] obtained by Padé approximant of the McMillan-Mayer perturbation expansion. The maximum appears at values of $z_{1}$ and $z_{2}$ outside of the experimentally investigated region [1]. The dependence of the maximum on $N_{2} / N_{1}$ predicted by equations (16) to (18) is different from that of the experimental results in [1]. Schäfer [6] pointed out that the maximum observed in [1] is the result of the treatment of the experimental data and not the feature of the true virial coefficient. The maximum of $A_{2}$ predicted by our theory would be difficult to observe experimentally because very large degrees of polymerization are needed.

\section{Comparison with the massless scheme.}

Since in the limit $N_{2} \rightarrow \infty$ and finite $N_{1} v_{22}(x)$ behaves like $S_{2}^{-\varepsilon / 2}$, the integral in (14) tends to zero and for $v_{12}$ follows

$$
v_{12}=v_{12}^{0}\left(S_{1}^{0} / S_{1}\right)\left(S_{2}^{0} / S_{2}\right) .
$$

This result is at variance with that obtained in $[7,8]$ by means of the massless schemes. As we have seen above, the expression for $v_{12}$ obtained in the $\Lambda^{2}$-scheme depends on the cutoff $\Lambda_{\min }$ and not on $S_{1}$ and $S_{2}$ and cannot be used without identification of $\Lambda_{\min }$ to study the limit of large $N_{2}$. The fifth diagram (made dimensionless) in figure 1 is regular for large $\mathrm{N}_{2}$ and its infrared behaviour is controlled by $N_{1}$. If it is assumed that the contribution to $A_{12}$ from the diagrams of the next orders remains finite in the limit of large $N_{2}$ the cutoff $\Lambda_{\min }$ has to be identified with $1 / S_{1}$ and for

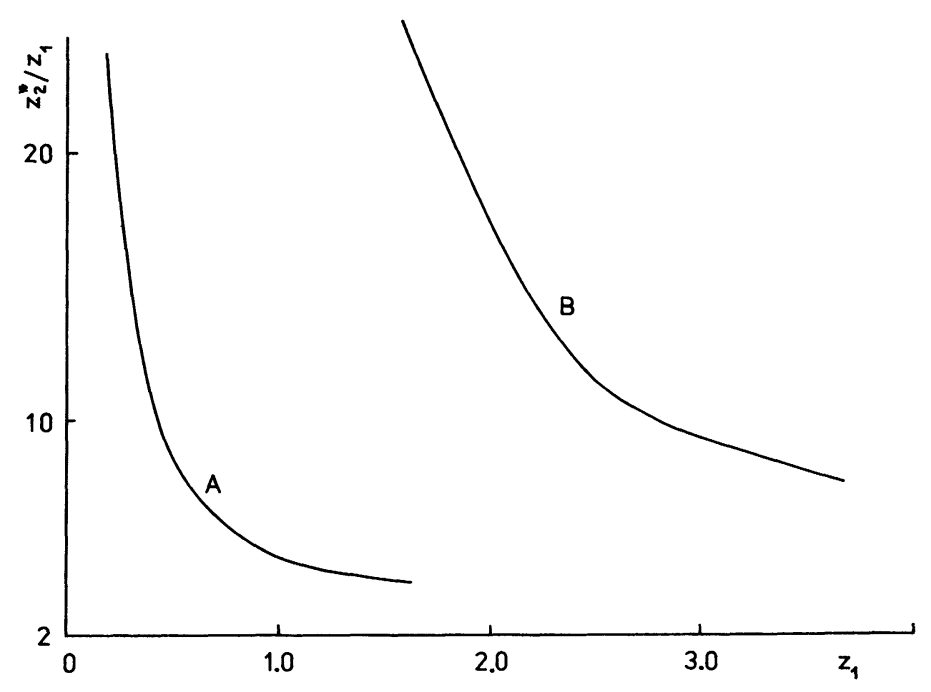

Fig. 2. - The lower bound above which the maximum of $A_{2}$ exists (A) Tanaka and Solc; (B) our work. 
$v_{12}$ it follows in the scaling region

$$
v_{12}=\left(v_{11} / v_{11}^{0}\right)^{1 / 2}\left(S_{1}^{0} / S_{1}\right)\left(S_{2}^{0} / S_{2}\right) .
$$

Since the mutual coefficient $A_{12}$ scales as $v_{12}\left(S_{1} / S_{1}^{0}\right)\left(S_{2} / S_{2}^{0}\right)$ it will be obtained for $A_{12}$

$$
A_{12} \approx\left(v_{11} / v_{11}^{0}\right)^{1 / 2} \approx N_{1}^{v d-2}
$$

the result which was obtained in [7, 8]. This result is caused by the assumption of the regularity of the many-loop diagrams. Really, the two-loop diagram in figure 1 diverges for $N_{2} \rightarrow \infty$ and consequently (22) is wrong. This can also be explained in the following way. The one-loop diagrams with one wave line between the chains (see Fig. 1) does not give a singular contribution (in four dimensions $v^{0} \ln \left(S^{0} \Lambda^{2}\right)$ ) to $A_{12}$. As a consequence of the $\mathrm{RG}$ invariance of the polymer theory the main logarithms of such types of diagrams of the next orders of the perturbation expansions do not contribute to $A_{12}$, too. The only one-loop diagram giving regular and singular contributions to $A_{12}$ is the fifth diagram in figure 1 . This diagram converges for $N_{2} \rightarrow \infty$. But the two-loop diagram in figure 1 diverges for $N_{2} \rightarrow \infty$. In this limit it decomposes in a product of the fifth diagram in figure 1 (its values we denote as $J$ ) and of the singular part of fourth diagram $\left(v_{22}^{0} \ln \left(S_{2}^{0} \Lambda^{2}\right)\right)$. Then, the contribution of the two last diagrams in figure 1 will be

$$
J\left(1-v_{22}^{0} \ln \left(S_{2}^{0} \Lambda^{2}\right)+\cdots\right) .
$$

The diagrams of the next orders extend the expression in the bracket in (23) to a series in powers of $v_{22}^{0} \ln \left(S_{2}^{0} \Lambda^{2}\right)$, which scales as $S_{2}^{0} / S_{2}$. So, for $N_{2} \rightarrow \infty$ and finite $N_{1}$ the contribution of the diagrams with two wave lines between the chains to $A_{12}$ goes to zero and may not produce the scaling law (22).

\section{Conclusion.}

The aim of the present work was to propose an RG method to describe the polydispersity effects in dilute polymer solutions. In the special case of polymer chains with different excluded volume strengths $v_{i j}^{0}$ but the same degree of polymerization, our method gives results compatible with previous investigations. When the degree of polymerization of one of the polymers becomes very large, the function $h_{12}$ tends to one and a maximum of the second virial coefficient takes place. These results are at variance with those obtained in [7, 8]. Our results are obtained in the oneloop approximation of the RG ( $\varepsilon$-results). The question of whether the next orders of the RG affect these results or not is not clear, and requires further investigation.

\section{Acknowledgments.}

Many thanks are due to Professor G. Helmis for stimulating discussions, to Professor L. Schäfer for helpful discussions during the Meco-Seminar at Gernrode. We also wish to thank the unknown referees whose criticism helped us to improve the paper, and Dr. J.-F. Joanny for sending us the reprint of the paper [8]. 


\section{References}

[1] Noda, I., Kitano, T., Nagasawa, M., J. Polym. Sci. Polym. Phys. Ed. 15 (1977) 1129.

[2] Elderfield, D., J. Phys. C 13 (1980) 5883.

[3] Nikomarov, E. S., Obuchov, S. P., JETP 80 (1981) 650.

[4] Stepanow, S., Acta Polymer. 32 (1981) 97.

[5] TANAKA, G., ŠolC, K., Macromolecules 15 (1982) 791.

[6] SCHÄFER, L., Macromolecules 15 (1982) 652.

[7] Witten, T. A., Prentis, J. J., J. Chem. Phys. 77 (1982) 4247.

[8] Joanny, J.-F., Leibler, L., Ball, R., J. Chem. Phys. 81 (1984) 4640.

[9] Kosmas, M. K., J. Physique Lett. 45 (1984) L-889.

[10] SCHÄFER, L., Macromolecules 17 (1984) 1357.

[11] Bogoliubov, N. N., ShIRKov, D. V., Introduction to the Theory of Quantized Fields (Nauka, Moscow) 1976.

[12] Stepanow, S., Ann. Phys. (Leipzig) 40 (1983) 301.

[13] Des Cloizeaux, J., J. Physique 36 (1975) 281.

[14] Des Cloizeaux, J., J. Physique Lett. 41 (1980) L-151.

[15] Des Cloizeaux, J., J. Physique 42 (1981) 635.

[16] Casassa, E. F., Polymer 1 (1960) 169. 